\title{
$\beta$-Catenin expression is associated with cell invasiveness in pancreatic cancer
}

\author{
Jin Niang Nan ${ }^{1}$, Ok Ran Kim${ }^{1}$, and Myung Ah Lee ${ }^{1,2}$
}

\begin{abstract}
${ }^{1}$ Cancer Research Institute, ${ }^{2}$ Division of Medical Oncology, Department of Internal Medicine, College of Medicine, Seoul St. Mary's Hospital, The Catholic University of Korea, Seoul, Korea
\end{abstract}

Background/Aims: This study was tried to determine the role of $\beta$-catenin in invasion in pancreatic cancer.

Methods: We analyzed cancer invasiveness according to $\beta$-catenin expression in pancreatic cancer cell line. We also investigated the change in cancer invasiveness when $\beta$-catenin expression was changed. To enhance $\beta$-catenin activity, we treated low $\beta$-catenin cancer cell line, PANC1, with Wnt-3a conditioned media and transected $\beta$-catenin. We also treated high $\beta$-catenin expressing cell line, $\mathrm{BxPC}_{3}$, with XAV939, $\beta$-catenin inhibitor and siRNA for $\beta$-catenin to inhibit $\beta$-catenin expression.

Results: The high $\beta$-catenin expressing cancer cell line, $\mathrm{BxPC}_{3}$ showed higher invasiveness, and low $\beta$-catenin expressing cell lines, PANCiand MIA-PaCa-2, were less invasive. By adding the Wnt-3a conditioned media or performing transfection with $\beta$-catenin in PANC1, cell invasiveness was increased $(p<0.05$ and $p<$ o.01, respectively). On inhibition of $\beta$-catenin by $\mathrm{XAV}_{939}$ and siRNA in $\mathrm{BxPC}_{3}$ cell line, invasiveness was significantly decreased $(p<0.01)$. It was not correlated with the expression of cluster of differentiation 44 (CD44) or CD44 variant 6 (CD44v6), the invasion related protein. On analysis of association with metastasis in human tissue, Wnt-3a expression was statistically correlated with the development of metastasis $(p=0.029)$.

Conclusions: Based on our data, $\beta$-catenin may be involved in cancer invasion in pancreatic cancer, and it is not associated with $\mathrm{CD}_{44}$, the invasion related protein.

Keywords: Pancreatic neoplasms; Beta-catenin; Invasiveness

\section{INTRODUCTION}

Pancreatic cancer is known to be an aggressive cancer with a 5-year survival rate of $8 \%[1]$. There are various factors related to high aggressiveness, such as aging characteristics, resistance to treatment, and early metastasis. Surgical resection is only curative treatment, but most patients are diagnosed when they are in unresectable state. Moreover, recurrences develop in $75 \%$ to $93 \%$ of patients, even in those who underwent surgery followed by adjuvant treatment $[2,3]$. Various molecular changes and responses in tumor and its microenvironment in pancreatic cancer can play a role in invasiveness, growth and chemoresistance, leading to highly aggressive tumor behavior. However, the mechanism of early metastasis in pancreatic cancer is not yet known.

Wnt/ $\beta$-catenin signaling plays an important role in many biological processes, including carcinogenesis and progression. In various solid tumors, activation of Wnt $/ \beta$-catenin signaling is known to be involved in invasion and metastasis. In colon cancer, it promoted scattering of cancer cells and metastasis by inhibition 
of the phosphatidylinositide 3-kinases-protein kinase B ( $\mathrm{PI}_{3} \mathrm{~K}-\mathrm{Akt}$ ) signaling pathway [4]. It also regulates metastasis by matrix metallopeptidase 9 (MMP-9) expression in bladder cancer [5]. Wnt/ß-catenin activation was observed in $65 \%$ of pancreatic adenocarcinomas, associated with invasion and metastasis or invasion [6-8]. Some researchers have suggested that the Wnt signaling pathway regulates migration, invasion, and metastasis in pancreatic cancer by producing pancreatic stellate cells [9]. As reported in previous studies, the Wnt/ $\beta$-catenin pathway can be directly or indirectly involved in the mechanism of metastasis.

In the present study, we tried to determine the role of the Wnt/ $\beta$-catenin signaling pathway by using pancreatic cancer cell lines and human tissue.

\section{METHODS}

\section{Cell lines}

We used pancreatic cancer cell lines including Panc-1, Mia-paca2, BxPC3, Capan2, which were obtained from American Type Culture Collection (ATCC; Manassas, VA, USA). They were maintained in Dulbecco's Modified Eagle Medium (DME; Hyclone, Logan, UT, USA) or Roswell Park Memorial Institute 1640 Medium (RPMI, Hyclone) containing 10\% fetal bovine serum (Hyclone) and $100 \mathrm{U} / \mathrm{mL}$ penicillin-streptomycin (Hyclone) maintained at $37^{\circ} \mathrm{C}$ in humidified air containing $5 \% \mathrm{CO}_{2}$. We performed the assessment of $\beta$-catenin expression in each cell line (Fig. 1A).

\section{Western blotting assay}

Cells were lysed in protein extraction buffer RIPA (Elpis
Biotech, Daejeon, Korea) with protein inhibitor cocktail (Roche, Berlin, Germany). Protein quantification was performed using BCA assay (Thermo Scientific, Rockford, IL, USA). Protein (5 to $20 \mu \mathrm{g} /$ lane) was sepa) rated by $6 \%$ to $10 \%$ SDS-PAGE and transferred to polye vinylidene difluoride membrane (PVDF, GE Healthcare Life Sciences, Freiburg, Germany). Blots were blocked with $5 \%$ skim milk solution and incubated with primary antibodies overnight at $4^{\circ} \mathrm{C}$. Next day, the blot was incubated with the secondary antibody for 1 hour, and was detected with ECL (Recenttec, Taipei, Taiwan). We used primary antibodies at a specific dilution; glyceraldehyde 3-phosphate dehydrogenase (GAPDH, Abcam, Cambridge, MA, USA) diluted 1:5,000, CD44v6 (Abcam) diluted 1:500, CD44 (GeneTex Inc., Irvine, CA, USA) diluted 1:500, $\beta$-catenin (ab6302, Abcam) diluted 1:4,000, and active- $\beta$-catenin (Merk Miillipore, Burlington, MA, USA) diluted 1:300.

\section{Cell invasion assay}

Cell invasion assays were conducted on $6.5 \mathrm{~mm}$ Transwell with $8.0 \mu \mathrm{m}$ pore size polycarbonate membrane insert. For the coating of invasion assay, matrigel (Corning) was diluted at a $1 / 20$ dilution rate with serum-free media and $100 \mu \mathrm{L}$ matrigel dilution buffer was coated onto the upper compartment of the cell culture insert. After incubation for 2 hours at $37^{\circ} \mathrm{C}$, the cell culture insert was ready for seeding. Pancreatic cancer cells (1.0 $\times 10^{5}$ to $2.0 \times 10^{5}$ ) were placed in matrigel-coated upper chambers with serum-free media for invasion assays and a medium containing $10 \%$ serum media was used as a chemoattractant in the lower chambers. After incubation at $37^{\circ} \mathrm{C}$, invaded cells were stained using DiffQuik staining kit (Sysmex, Kobe, Japan). The images of cells
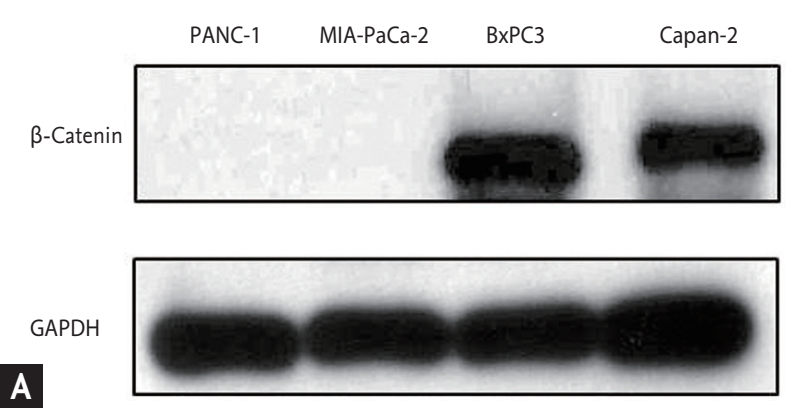

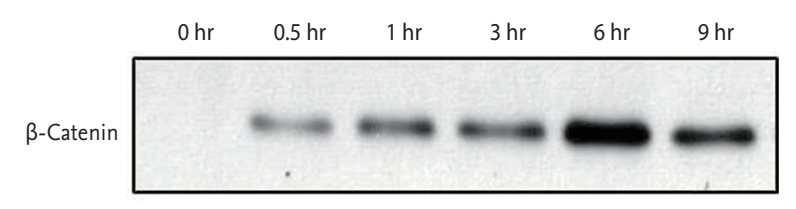

GAPDH

\section{B}

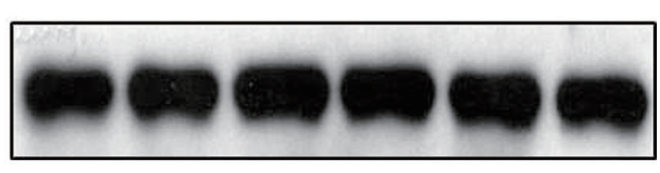

Figure 1. Expression of $\beta$-catenin. (A) Expression of $\beta$-catenin in each pancreatic cancer cell lines. (B) After Wnt-za conditioned media, $\beta$-catenin expression was significantly increased and maximum at 6 hours. GAPDH, glyceraldehyde 3-phosphate dehydrogenase. 
were photographed with EVOS Cell Imaging Systems (Left Technologies, Gaithersburg, MD, USA) at $\times 200$ magnification and the cell number was counted in three random fields of view.

\section{Activation of $\beta$-catenin}

\section{Application of Wnt-3a conditioned media}

We collected media from Wnt-za-expressing L cells and cultured media and control media from mouse L cells, prepared according to the manufacturer's instructions (ATCC). We tested the Wnt-za conditioned media by $\beta$-catenin expression using Western blot. $\beta$-Catenin expression was maximum at 6 hours after Wnt-3a conditioned media (Fig. 1B); therefore, we maintained Wnt-3a stimulation by changing the media every 6 hours.

\section{Transfection of $\beta$-catenin}

For stable expression of $\beta$-catenin, we used $\beta$-catenin pcDNA3 $_{3}$ (Plasmid 16828\#, Addgene, Cambridge, MA, USA), pcDNA3-S33Y $\beta$-catenin (Plasmid \#19286, Addgene), the mutant form and $\mathrm{pcDNA}_{3}$ (Invitrogen, USA) as the control vector. The PANC1 cell line, non $\beta$-catenin expressing cell line, was seeded in 6-well dishes and then $\beta$-catenin was transfected using Lipofectamine 2000 (Thermo Fisher, San Jose, CA, USA). After 48 hours, the cells were harvested and Western blotting assays were performed.

\section{Inhibition of $\beta$-catenin}

XAV939 treatment

XAV939 (Selleckchem, Houston, TX, USA), a Wnt/ $\beta$-catenin signaling inhibitor, was diluted to a final concen- tration of $1 \mu \mathrm{M}$, and then it was applied to the $\mathrm{BxPC}_{3}$ cell line. We harvested co-cultured cells and performed Western blot.

\section{Application of siRNA for $\beta$-catenin}

The $\mathrm{BxPC}_{3}$ cell line was cultured in a 6-well plate, and it was treated with $20 \mathrm{nmol} / \mathrm{L}$ control siRNA (Sc37007; Santa Cruz) and $\beta$-catenin siRNA (Sc292009; Santa Cruz) by adding TransIT-TKO Transfection Reagent MIR 2150 (Mirus, Madison, WI, USA). The siRNAs were cultured for 48 hours and then cell lysates were subsequently prepared for Western blot to verify knockdown of $\beta$-catenin.

\section{Tissue microarray and immunohistochemical staining} Paraffin blocks with tumor samples were obtained from 73 patients who had undergone curative resection for pancreatic cancer between January, 2001 and December, 2009. Tissue microarray was constructed as mentioned previously [10]. We performed immunohistochemical staining with the following primary antibodies at specified dilutions: Wnt-3a (Abcam) diluted 1:100, Wnt5a (Abcam) diluted 1:50, and $\beta$-catenin (Abcam) diluted 1:100. The results were interpreted by two independent pathologists who were blinded to the specific diagnosis and prognosis of each case. The staining intensity was scored on a three-tiered scale: score 0 , less than $10 \%$ of cells positive; $1,10 \%$ to $49 \%$ positive; and 2 , more than $50 \%$ of cells positive. We also reviewed the patients' medical records to analyze the clinical outcome according to Wnt/ß-catenin expression. This study was approved by our Institutional Review of Board: Board in Seoul St. Mary's Hospital (KC11SISIo184).
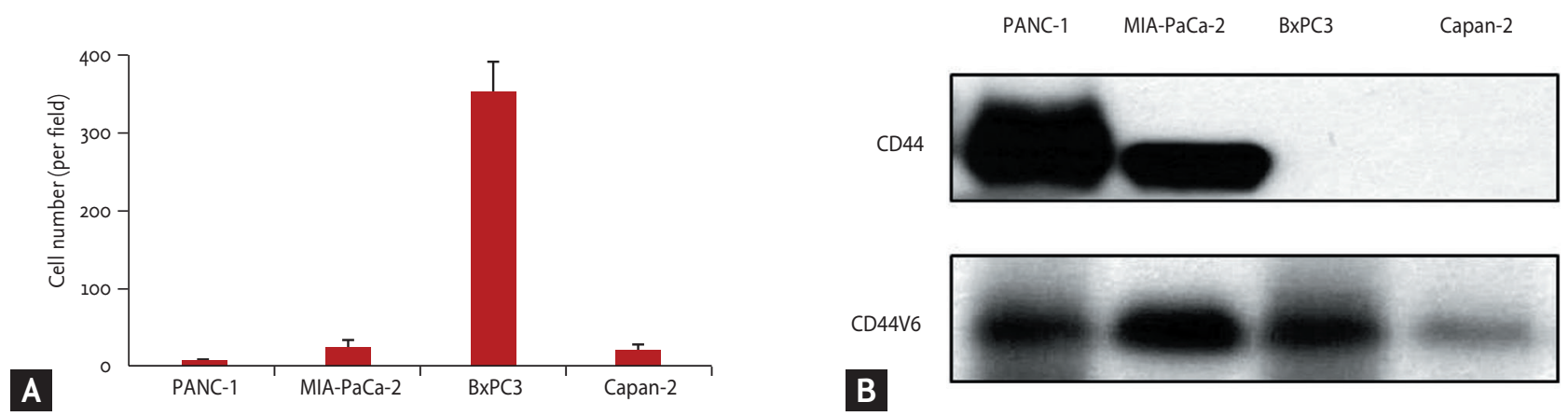

Figure 2. (A, B) Transwell invasion assay. The pancreatic cell was most invasiveness in BxPC3 cell line, not associated with cluster of differentiation 44 (CD44) or CD44 variant 6 (CD44v6) expression. 


\section{Statistical analysis}

All data are presented as mean \pm standard deviation $(\mathrm{SD})$ of at least three separate experiments. Student $t$ tests were used for transwell invasion assay. The chi-square and Fisher exact tests were used to analyse correlations between immunohistochemical profiles and clinicopathologic variables. Kaplan-Meier tests were used to analyse the survival data. Statistical significance was set at $p<0.05$. Statistical analyses were performed using the IBM SPSS version 24.0 (IBM Corp., Armonk, NY, USA).

\section{RESULTS}

\section{Cell invasion assay}

On Transwell invasion assay, the $\mathrm{BxPC}_{3}$ cell line, high $\beta$-catenin expressing cell line was the most invasive, and Panc-1, the non $\beta$-catenin expressing cell line showed weak invasiveness. It was not associated with expression of $\mathrm{CD}_{44}$ or $\mathrm{CD} 44$ variant, known as the invasion related protein (Fig. 2). We chose $\mathrm{BxPC}_{3}$ and Panc-1 cell lines for further experiments as $\beta$-catenin expressing and non $\beta$-catenin expressing cell lines, respectively.

\section{Over expression of $\beta$-catenin enhanced cell inva- siveness}

The Panc-1, the low $\beta$-catenin expressing cell line was incubated in Wnt-3a conditioned media and then tested with the cell invasion assay. Invasion was increased after Wnt-3a conditioned media treatment of Panc-1 cell line, low $\beta$-catenin expressing cell line CM $(p<0.05)$. During treatment with Wnt-3a conditioned media, CD44 or CD44v6 expression was not changed (Fig. 3A). After transfection of $\beta$-catenin into Panc-1 cell line, cell invasion was increased, after transfection of both wildtype and mutant $\beta$-catenin $(p=0.01$ ) (Fig. 3B). It was not associated with $\mathrm{CD}_{44}$ and $\mathrm{CD} 44 \mathrm{v} 6$ expression.

\section{Inhibition of $\boldsymbol{\beta}$-catenin reduced cell invasiveness}

When the $\mathrm{BxPC} 2$ cell line was treated with $\mathrm{XAV}_{939}$, activated $\beta$-catenin expression was reduced and invasiveness was also decreased $(p<0.01)$ (Fig. $4 \mathrm{~A})$. The same
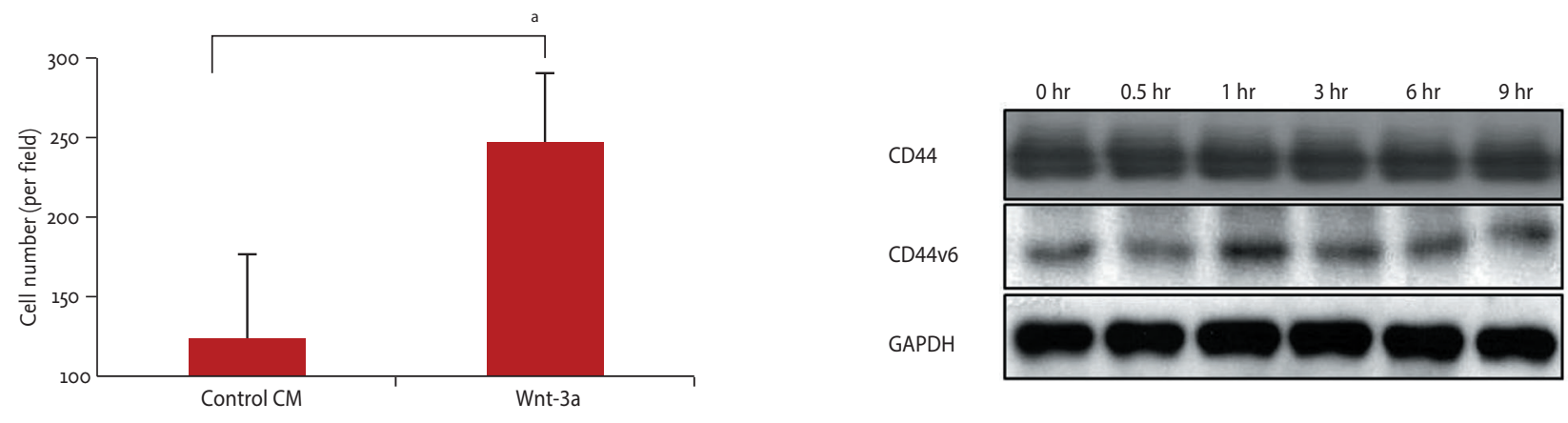

A
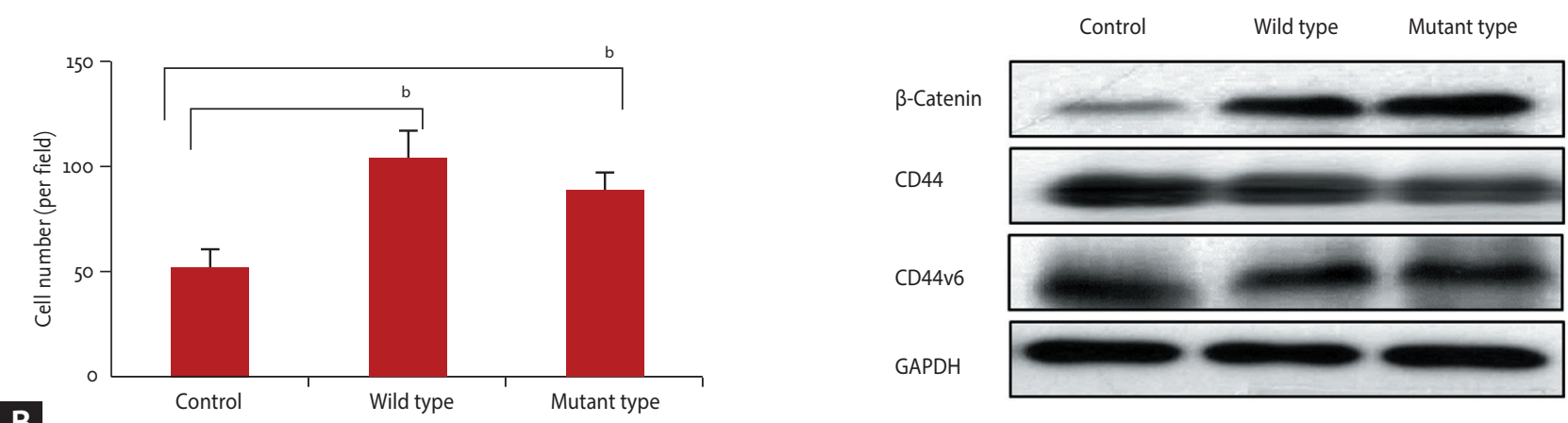

B

Figure 3. Change of cell invasion after $\beta$-catenin activation. (A) The cell invasion was increased without change of cluster of differentiation 44 (CD44) or CD44 variant 6 (CD44v6) expression after Wnt-3a media treatment. (B) The cell invasion was also increased after transfection $\beta$-catenin both wild and mutant $\mathrm{S}_{33} \mathrm{Y}$ type without dependent CD44 or CD44V6 expression. GAP$\mathrm{DH}$, glyceraldehyde 3-phosphate dehydrogenase. ${ }^{\mathrm{a}} \mathrm{p}<0.05,{ }^{\mathrm{b}} \mathrm{p}<0.01$. 
result was found when $\beta$-catenin expression was interfered with $\beta$-catenin siRNA in $\mathrm{BxPC}_{3}$ cell line $(p<0.01)$ (Fig. 4B).

\section{Immunohistochemical staining in human tissue}

We analyzed the association between Wnt protein expression and the presence of lymph node or development of organ metastasis in the follow-up period. There was no significant correlation with initial stage or lymph node involvement, but Wnt-3a expression was correlated with development of distant organ metastasis after curative surgery $(p=0.029)$ in the follow-up period (Table 1). However, there was no difference in survival outcome according to Wnt expression $(p=0.703, p=0.363$, and $p$ $=0.401$ for $\beta$-catenin, Wnt3-a, and Wnt-5a, respectively) (Fig. 5).
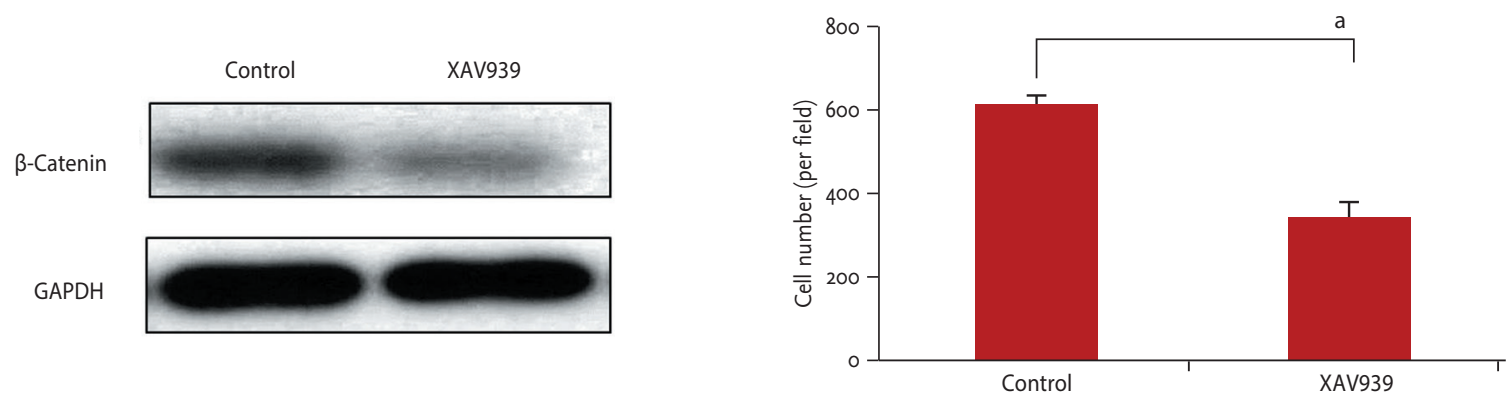

\section{A}

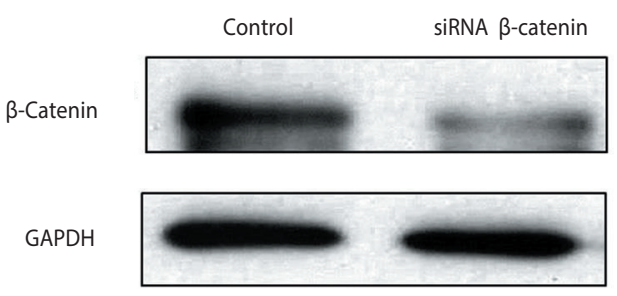

B

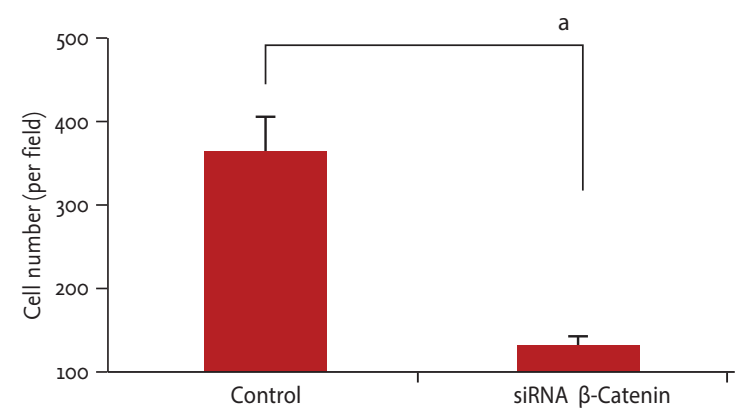

Figure 4. Change of cell invasion after $\beta$-catenin inhibition. (A) The cell invasion was decreased after $\beta$-catenin expression inhibition by XAV939 (Wnt/ $\beta$-catenin signaling inhibitor). (B) The cell invasion was also decreased after treatment of siRNA for $\beta$-catenin. GAPDH, glyceraldehyde 3-phosphate dehydrogenase. ${ }^{\mathrm{a}} \mathrm{p}<0.01$.

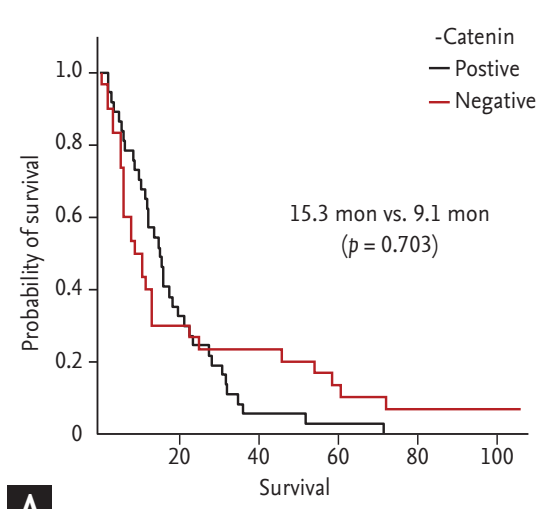

A

Figure 5. Survival outcome according to Wnt expression. (A) Overall survival for $\beta$-catenin (15.3 months vs. 9.1 months, $p=$ 0.703). (B) Overall survival for Wnt-3a (12.3 months vs. 8.2 months, $p=0.363)$. (C) Overall survival for Wnt-5a (12.4 months vs.9.1 months, $p=0.401$ ). 
Table 1. Wnt expression and its association between the clinical findings

\begin{tabular}{|c|c|c|c|c|c|c|c|c|c|}
\hline \multirow{2}{*}{ Variable } & \multicolumn{3}{|c|}{$\beta$-Catenin } & \multicolumn{3}{|c|}{ Wnt-3a } & \multicolumn{3}{|c|}{ Wnt-5a } \\
\hline & + & - & $p$ value & + & - & $p$ value & + & - & $p$ value \\
\hline $\mathrm{T} 1$ & 3 & 1 & 0.635 & 4 & 0 & 0.223 & 4 & 0 & 0.424 \\
\hline $\mathrm{T} 2$ & 10 & 12 & & 18 & 5 & & 21 & 2 & \\
\hline $\mathrm{T}_{3}$ & 15 & 10 & & 15 & 55 & & 21 & 5 & \\
\hline $\mathrm{T}_{4}$ & 11 & 9 & & 15 & 6 & & 16 & 5 & \\
\hline No & 17 & 11 & 0.293 & 21 & 31 & 0.191 & 26 & 3 & 0.221 \\
\hline N1 & 22 & 21 & & 31 & 14 & & 36 & 9 & \\
\hline Metastasis + & 26 & 20 & 0.453 & 29 & 18 & $0.029^{\mathrm{a}}$ & 38 & 9 & 0.288 \\
\hline Metastasis - & 13 & 12 & & 23 & 4 & & 24 & 3 & \\
\hline
\end{tabular}

${ }^{a}$ Wnt-3a expression showed significant correlation with development of metastasis.

\section{DISCUSSION}

Pancreatic cancer is known to be a highly aggressive cancer because of early metastasis, leading to death; however, its mechanism is still not understood. The $\mathrm{K}$-ras mutation is commonly found in $90 \%$ of pancreatic cancers and it can activate the Wnt/ $/$-catenin signaling pathway, involved in up-regulation of epithelial mesenchymal transition stimulation [11]. In previous studies, it was reported that $\mathrm{Wnt} / \beta$-catenin may play a role in cancer invasion or metastasis in hepatocellular cancer and biliary tract cancer [12,13].

In the present study, we tried to determine the association between Wnt/ $\beta$-catenin and cell invasion. We also investigated the expression of $\mathrm{CD}_{44}$ and $\mathrm{CD}_{44}$ variant, well-known invasion related proteins in pancreatic cancer to identify the relation with $\mathrm{Wnt} / \beta$-catenin. The $\beta$-catenin expressing cell line, $\mathrm{BxPC}_{3}$ cell line, showed high invasiveness compared to two $\beta$-catenin non-expressing cell lines. These two $\beta$-catenin non-expressing cell lines were not invasive even with high expression of $\mathrm{CD}_{44}$ and $\mathrm{CD}_{44}$ variant. We enhanced or suppressed $\beta$-catenin expression in each cell line to determine if the change in $\beta$-catenin expression affects cell invasion. On adding the Wnt-3a conditioned media to the low $\beta$-catenin expressing cell line; $\beta$-catenin expression was transiently increased, as shown in Fig. 1. When $\beta$-catenin expression was increased, cell invasion of PANC1 was increased, without any change in the $\mathrm{CD}_{44}$ or $\mathrm{CD}_{44}$ variant expression. $\beta$-Catenin was transfected into the PANC1 cell line for producing a transient $\beta$-catenin expressing cell line. We used wild-type and activated $\beta$-catenin to determine if the type of $\beta$-catenin plays a specific role in cell invasion. In our study, the type of $\beta$-catenin did not play a critical role in invasion. It was also not associated with $\mathrm{CD}_{44}$ or $\mathrm{CD}_{44}$ variant expression. Considering that both types of $\beta$-catenin expression significantly increased cell invasiveness, Wnt $/ \beta$-catenin activation itself can be associated with cell invasion and metastasis, but it may not be associated with $\mathrm{CD}_{44}$ activation.

XAV939, a potent tankyrase (TNKS) inhibitor, deregulates the Wnt/ $\beta$-catenin signaling pathway in various cancers by stabilizing axin, without affecting nuclear factor kappa B or transforming growth factor- $\beta$ [14]. We treated $\mathrm{BxPC}_{3}$ cell line with $\mathrm{XAV}_{939}$ to inhibit $\beta$-catenin activity. As shown in Fig. 4, cell invasiveness was significantly decreased during $\beta$-catenin expression. A similar result was found in our experiment with siRNA for $\beta$-catenin. All these data indicated that the Wnt $/ \beta$-catenin signaling pathway can be involved in cell invasion and metastasis in pancreatic cancer.

In a previous study, the Wnt/ $\beta$-catenin signal pathway was found to be involved in cancer invasion and metastasis through various mechanisms in solid tumor [5,15]. Pai et al. [7] suggested that the aberrant Wnt signaling pathway contributed to progression of pancreatic cancer by promoting mucin 4 (MUC4). In one report, inhibition of Wnt $/ \beta$-catenin caused regression of tumor growth in a mouse model [16]. However, we investigated the role of $\beta$-catenin by direct enhancement or inhibition of $\beta$-catenin, without using the Wnt signaling pathway related protein unlike the previous trials. This suggested that the target for $\beta$-catenin, not for any step of the 
Wnt/ $\beta$-catenin effector pathway can control invasion or metastasis in pancreatic cancer.

We also investigated the association between Wnt protein expression and the development of metastasis in human tissue. In our study, Wnt-3a protein expression showed a correlation with metastasis, but not $\beta$-catenin. First, the Wnt signaling pathway has been known to be activated by two pathways, $\beta$-catenin dependent pathway (canonical pathway) and $\beta$-catenin independent pathway (non-canonical pathway), and the Wnt-3a, in the upper stream, activates both pathways [17]. In the previous study, the non-canonical pathway as well as the canonical pathway was related to cell growth, migration, or metastasis [18-20]. The Wnt-3a may regulate cancer invasion or metastasis through non-canonical pathway, independent of $\beta$-catenin. Second, we analyzed a small number of patients with different stages in the present study; thus, we could not adequately confirm the in vitro data.

There are some limitations to our study; therefore, our results should be interpreted with caution. First, we performed the experiments at a cell line level, and therefore, an in vivo test is required to support our hypothesis. Second, we did not confirm any interaction with other metastasis related protein except CD44. Therefore, we could not explain how Wnt $/ \beta$-catenin acts on metastasis. The Wnt/ $\beta$-catenin signaling pathway can interact with many signaling pathways for tumor and tumor microenvironment as a "controller of the signaling pathway." To define the mechanism for metastasis in pancreatic cancer, we should perform further research considering this point.

In spite of these limitations, our research is worthy as it provides information about the potential role of Wnt $/ \beta$-catenin associated with metastasis in pancreatic cancer. We also observed the same result in human tissue, and not only in cell line. Based on our result, Wnt $/ \beta$-catenin can be another potential target to control the progression in pancreatic cancer.

\section{KEY MESSAGE}

1. The Wnt $/ \beta$-catenin signaling pathway can be involved in the cancer invasion in pancreatic cancer.
2. It should not be associated with invasion related protein, cluster of differentiation 44 (CD44) or CD 44 variant.

\section{Conflict of interest}

No potential conflict of interest relevant to this article was reported.

\section{REFERENCES}

1. Siegel RL, Miller KD, Jemal A. Cancer statistics, 2016. CA Cancer J Clin 2016;66:7-30.

2. Sugiura T, Uesaka K, Mihara K, et al. Margin status, recurrence pattern, and prognosis after resection of pancreatic cancer. Surgery 2013;154:1078-1086.

3. Oettle H, Neuhaus P, Hochhaus A, et al. Adjuvant chemotherapy with gemcitabine and long-term outcomes among patients with resected pancreatic cancer: the CONKO-oo1 randomized trial. JAMA 2013;310:1473-1481.

4. Tenbaum SP, Ordonez-Moran P, Puig I, et al. $\beta$-Catenin confers resistance to $\mathrm{PI}_{3} \mathrm{~K}$ and AKT inhibitors and subverts $\mathrm{FOXO}_{3}$ a to promote metastasis in colon cancer. Nat Med 2012;18:892-901.

5. Du Y, Wang Y, Zhang F, et al. Regulation of metastasis of bladder cancer cells through the WNT signaling pathway. Tumour Biol 2015;36:8839-8844.

6. Zeng G, Germinaro M, Micsenyi A, et al. Aberrant Wnt/ beta-catenin signaling in pancreatic adenocarcinoma. Neoplasia 2006;8:279-289.

7. Pai P, Rachagani S, Lakshmanan I, et al. The canonical Wnt pathway regulates the metastasis-promoting mucin MUC4 in pancreatic ductal adenocarcinoma. Mol Oncol 2016;10:224-239.

8. Arensman MD, Kovochich AN, Kulikauskas RM, et al. $\mathrm{WNT}_{7} \mathrm{~B}$ mediates autocrine Wnt/ $\beta$-catenin signaling and anchorage-independent growth in pancreatic adenocarcinoma. Oncogene 2014;33:899-908.

9. Xu Y, Li H, Huang C, et al. Wnt2 protein plays a role in the progression of pancreatic cancer promoted by pancreatic stellate cells. Med Oncol 2015;32:97.

10. Ko YH, Cho YS, Won HS, et al. Prognostic significance of autophagy-related protein expression in resected pancreatic ductal adenocarcinoma. Pancreas 2013;42:829-835. 
11. Xu W, Wang Z, Zhang W, et al. Mutated K-ras activates CDK8 to stimulate the epithelial-to-mesenchymal transition in pancreatic cancer in part via the $\mathrm{Wnt} / \beta$-catenin signaling pathway. Cancer Lett 2015;356(2 Pt B):613-627.

12. Yun KJ, Han WC, Choi SC, Kim TH. Immunohistochemical study of beta-catenin expression between hepatocellular carcinoma and cholangiocarcinoma. Cancer Res Treat 2002;34:117-211.

13. Moon WS, Park HS, Lee H, et al. Co-expression of cox-2, $\mathrm{C}$-met and beta-catenin in cells forming invasive front of gallbladder cancer. Cancer Res Treat 2005;37:171-176.

14. Huang SM, Mishina YM, Liu S, et al. Tankyrase inhibition stabilizes axin and antagonizes Wnt signalling. Nature 2009;461:614-620.

15. Lee MA, Park JH, Rhyu SY, Oh ST, Kang WK, Kim HN. Wntza expression is associated with MMP-9 expression in primary tumor and metastatic site in recurrent or stage IV colorectal cancer. BMC Cancer 2014;14:125.

16. Garg B, Giri B, Majumder K, Dudeja V, Banerjee S, Saluja A. Modulation of post-translational modifications in $\beta$-catenin and LRP6 inhibits Wnt signaling pathway in pancreatic cancer. Cancer Lett 2017;388:64-72.

17. Qiang YW, Endo Y, Rubin JS, Rudikoff S. Wnt signaling in B-cell neoplasia. Oncogene 2003;22:1536-1545.

18. Jessen JR. Noncanonical Wnt signaling in tumor progression and metastasis. Zebrafish 2009;6:21-28.

19. Tao YM, Liu Z, Liu HL. Dickkopf-1 (DKKı) promotes invasion and metastasis of hepatocellular carcinoma. Dig Liver Dis 2013;45:251-257.

20. Kurayoshi M, Oue N, Yamamoto H, et al. Expression of Wnt-5a is correlated with aggressiveness of gastric cancer by stimulating cell migration and invasion. Cancer Res 2006;66:10439-10448. 